\title{
The clinical effect and relevant mechanism of combined sorafenib and radiofrequency ablation in the treatment of early small hepatocellular carcinoma
}

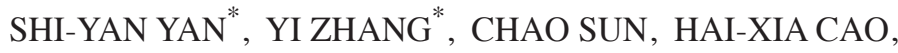 \\ GUANG-MING LI, YU-QIN WANG and JIAN-GAO FAN \\ Department of Gastroenterology, Xinhua Hospital, Shanghai Jiaotong \\ University School of Medicine, Shanghai 200092, P.R. China
}

Received May 7, 2016; Accepted June 9, 2016

DOI: $10.3892 / \mathrm{ol} .2016 .4694$

\begin{abstract}
The number of cases with hepatocellular carcinoma (HCC) are on the increase. The aim of the present study was to investigate the clinical effect and relevant mechanism of combined sorafenib and radiofrequency ablation (RFA) in the treatment of the early small HCC. A total of 120 cases of patients with small HCC that presented during the period of May 2007 to June 2010 were selected and divided into the surgery $(n=60)$ and RF $(n=60)$ groups according to the treatment method employed. The surgery group was treated with a laparotomy resection and the RF group was treated with combined sorafenib and RFA, and a comparative analysis was made between the two groups with regard to recurrence rates, adverse reactions, and survival rates. After treatment of 1 month, the radical effective rate of the surgery and RF groups was $100 \%$. Contrast-enhanced ultrasound images of the patients in the RF group were taken. During the 5-year follow-up, the tumor recurrence rate in the surgery group was $18.3 \%$, significantly lower than that in the RF group where the tumor recurrence rate was $38.3 \%(\mathrm{P}<0.05)$. The occurrence rate of postoperative pain, fever, abdominal bleeding, infection, and other complications of patients in the surgery group was significantly higher than the complication occurrence rate $(\mathrm{P}<0.05)$ of the patients in the RF group. The average survival time of the patients in the surgery group was $51.2 \pm 1.5$ months and the survival rates during the first, third and fifth year were $90.7,71.5$ and $56.7 \%$, respectively. Additionally, the
\end{abstract}

Correspondence to: Drs Yu-Qin Wang or Jian-Gao Fan, Department of Gastroenterology, Xinhua Hospital, Shanghai Jiaotong University School of Medicine, 1665 Kongjiang Road, Shanghai 200092, P.R. China

E-mail: yu_qinwang1@163.com

E-mail: jian_gaofan1@163.com

*Contributed equally

Key words: sorafenib, radiofrequency ablation, small hepatocellular carcinoma, hepatectomy, clinical effect average survival time of the patients in the RF group was $64.6 \pm 2.4$ months and the survival rates during the first, third and fifth year were 91.1, 72.8 and $57.5 \%$, respectively. The difference between the two groups was not statistically significant. The tumor-free survival rates in the surgery group during the first, third and fifth year were 87.8, 44.3 and $33.2 \%$, respectively, while the tumor-free survival rates in the RF group during the first, third and fifth year were 86.2, 48.3 and $34.6 \%$, respectively, and the difference between the two groups was not statistically significant. In conclusion, the combined sorafenib and RFA method, and laparotomy resection method have their advantages in the treatment of early small HCC, and under specific medical conditions, the former can partially replace the latter and be used as a preferred treatment means in the treatment of early small HCC.

\section{Introduction}

The number of cases with primary hepatocellular carcinoma (HCC) has been on the increase in recent years Theincidence has reached to 250,000 new cases per year $(1,2)$. the mortality was relatively high and the five-year survivalrate was as low as $5 \%$ (1). Males were diagnosed more oftenthan females (5:1). Males over 40 and females 0ver 60 are the most affected population (2). Small HCC refers to a cancer nodule with an isolated tumor diameter of $\leq 3 \mathrm{~cm}$ clinically or a type of HCC with the sum of the diameters of the tumors on two adjacent nodules at $\leq 3 \mathrm{~cm}$ (1).

Laparotomy is currently recognized as an important method for the treatment of early small HCC, although such treatment schemes lead to relatively large body trauma (3). Radiofrequency ablation (RFA), a minimally invasive surgery employed in recent years, has caused increasing concern and demand on the medical profession in the clinical treatment of malignant tumors $(4,5)$. Sorafenib belongs to a multi-kinase inhibitor that blocks the generation of new blood vessels in the body of cancer patients and effectively inhibits cancer cell growth and proliferation. Previous findings $(6,7)$ confirmed that sorafenib improved the prognosis of advanced HCC, but, to the best of our knowledge, the clinical effect in the treatment of the early small HCC has rarely been reported. 
The aim of the present study was to investigate the clinical effect of combined sorafenib and RFA in the treatment of early small HCC, compared with the clinical effect of laparotomy resection and subsequently contrast the difference between the two treatment methods to assess their usefulness in the clinic.

\section{Patients and methods}

General information. In total, 120 patients (134 lesions) with early small HCC admitted to the Xinhua Hospital (Shanghai, China) between May 2007 and June 2010 were selected and included in the study. The enrolled cases were patients initially admitted and treated in the hospital, including 68 men and 52 women, aged 45-71 years, with a mean age of $62.6 \pm 2.5$ years. Concerning clinical stages, there were 64 and 56 cases at Ia and Ib stages, respectively, and all the cases were consistent with the clinical diagnostic criteria for primary HCC and confirmed by pathology. Simultaneously the color Doppler ultrasound examination identified a tumor size $\leq 3 \mathrm{~cm}$.

Inclusion criteria for the study were: i) Patients aged 45-71 years, primarily diagnosed as early small HCC, ii) patients in exhibiting tumors as per the 2001 Hepatocellular Carcinoma Clinical Diagnostic Criteria and Clinical Stage, iii) patients with a tumor diameter of $\leq 3 \mathrm{~cm}$, iv) patients without angiogenesis, bile duct violation, or distant metastasis; v) patients with liver function grading: Child-Pugh A or B, and vi) patients who signed the written informed consent form and agreed to undergo the treatment employed in the study. Patients with a liver function of Child-Pugh $\mathrm{C}$ grade, serious function failure of other vital organs and communication disorders and those not willing to participate in the study were excluded.

The 120 enrolled patients with small HCC (134 lesions) were divided into the surgery $(n=60)$ and $R F(n=60)$ groups in accordance with the treatment method. There were 60 cases including 67 lesions in the surgery and RF groups, respectively.

Approval for the study was obtained by the ethics committee of Xinhua Hospital.

Methods. The surgery group received laparotomy resection treatment under general anesthesia.

Combined sorafenib and RFA were used to treat the RF group. The percutaneous microwave ablation method was conducted as follows. Briefly, the microwave therapy apparatus was purchased from the Baoxing Medical Equipment Co., Ltd. (Jiangsu, China). The microwave operating frequency was set at $915 \mathrm{MHz}$, the output operation power was set at 30-50 W, and the transmission mode was set to pulse. Prior to accepting microwave ablation by irradiation, the patients underwent blood routine examination, liver function, prothrombin time, and other indicators. The patients were fasted on the date treatment was administered, while simultaneously, the intravenous accesses were established prior to treatment. The patients were generally treated under intravenous anesthesia. Following localization of the lesion using an ultrasonic instrument (Dynaflox Shanghai Tech Co., Ltd., Shanghai, China), the electrode was sent to the lesion site to be punctured under the guidance of contrast-enhanced ultrasonography (CEUS), and $5 \mathrm{~mm}$ was routinely cut around the tumor for the placement of the temperature measuring needle. Microwave power, treatment time, and other combinations were set according to the size of the lesion tissue, and the microwave ablation stop indication occurred when the temperature around the tumor was at $60^{\circ} \mathrm{C}$ and reached $54^{\circ} \mathrm{C}$ for a duration of $3 \mathrm{~min}$. The case treatment time of each group was $\sim 5-10 \mathrm{~min}$. The entire operation was performed under ultrasound dynamic and continuous monitoring. The electrocardiograph monitoring and oxygen situation of the patients were observed during the treatment and changes in the heart rate and blood pressure of the patients were strictly monitored. Of the 60 patients in the surgery group, 48 cases underwent ablation once, 10 cases underwent ablation twice, and 2 cases underwent ablation three times.

The sorafenib treatment method was carried out by treating patients in the RF group with additional sorafenib based on the abovementioned percutaneous microwave ablation method, i.e., $0.4 \mathrm{~g}$ of sorafenib was administered [Bayer Schering Pharma AG (Berlin, Germany), approval no. H20130137] each time: twice daily, orally $2 \mathrm{~h}$ after a meal, for 4 weeks.

Efficacy evaluation. After 1 month of treatment, all the cases underwent enhanced CT, $\alpha$-fetoprotein (AFP), and other examinations to evaluate the clinical efficacy, and a detailed record was made on each follow-up situation. If a patient was found with a new lesion versus the primary lesion or elevated AFP during the follow-up, it was considered as a tumor recurrence following treatment (8).

Statistical analysis. Data were statistically analyzed using SPSS 20.0 software (SPSS, Inc., Chicago, IL, USA). Comparison between groups was made with the t-test or Chi-square test. The overall survival and tumor-free survival rates were calculated using the Kaplan-Meier calculation method and analyzed with the log-rank test. A multifactor prognosis analysis was performed using the Cox proportional hazard model to obtain the independent risk factors affecting prognosis. $\mathrm{P}<0.05$ was considered to indicate a statistically significant difference.

\section{Results}

Comparison of the short-term efficacy and follow-up recurrence of the patients between the groups. After 1 month of treatment, the radical effective rate of the surgery and RF groups was $100 \%$. The contrast-enhanced ultrasound image of the patient in the RF group prior to and after treatment is shown in Figs. 1 and 2. During the 5-year follow up, the tumor recurrence rate of the surgery group was $18.3 \%$, significantly lower than the tumor recurrence rate in the RF group which was $38.3 \%(\mathrm{P}<0.05)$ (Table I).

Comparison of complications between the groups. The occurrence rates of the postoperative pain, fever, abdominal bleeding, infection, and other complications of patients in the surgery group were significantly higher than the complication incidence rate $(\mathrm{P}<0.05)$ of the patients in the RF group (Table II).

Comparison of the survival rates between the groups. The average survival time of the patients in the surgery group was $51.2 \pm 1.5$ months, and the survival rates during the first, third and fifth year were 90.7, 71.5 and $56.7 \%$, respectively. The average survival time of the patients in the RF group was $64.6 \pm 2.4$ months, and the survival rates during the first, 
Table I. Comparison of tumor follow-up recurrence between the two groups.

\begin{tabular}{lccccc}
\hline Group category & No. & Local recurrence & Distant recurrence & Distant recurrence & Recurrence rate, \% \\
\hline Surgery & 60 & 2 & 9 & 0 & 18.3 \\
RF & 60 & 7 & 12 & 4 & 38.3 \\
\hline
\end{tabular}

$\mathrm{RF}$, radiofrequency.

Table II. Comparison of adverse reactions between the two groups.

\begin{tabular}{lllll}
\hline Group category & No. & Pain $(\%)$ & Fever $(\%)$ & Abdominal bleeding (\%) \\
\hline Surgery & 60 & $38(63.3)$ & $29(48.3)$ & $7(11.7)$ \\
RF & 60 & $14(23.3)$ & $15(25)$ & $2(3.3)$ \\
\hline
\end{tabular}

RF, radiofrequency.

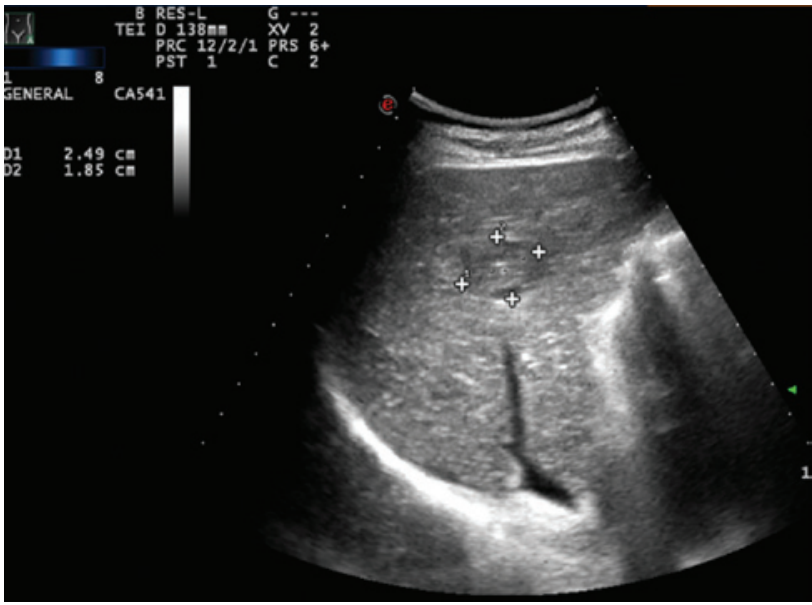

Figure 1. Contrast-enhanced ultrasound image for small hepatocellular carcinoma before treatment in the radiofrequency group.

third and fifth year were 91.1, 72.8 and $57.5 \%$, respectively. The difference between the two groups was not statistically significant (Fig. 3).

The tumor-free survival rates in the surgery group during the first, third and fifth year were $87.8,44.3$ and $33.2 \%$, respectively. The tumor-free survival rates in the RF group during the first, third and fifth year were 86.2, 48.3 and $34.6 \%$, respectively. The difference between the two groups was not statistically significant (Fig. 4).

\section{Discussion}

RFA treatment has attracted much attention from the medical community in the treatment of neoplastic diseases. Treatments including minimal invasive surgery and simple operation are currently considered viable HCC intervention treatment methods with improved prospects $(9,10)$. Previous studies have reported that (9-11), on the one hand, RFA can achieve necrosis of cancer cells by directly coagulating

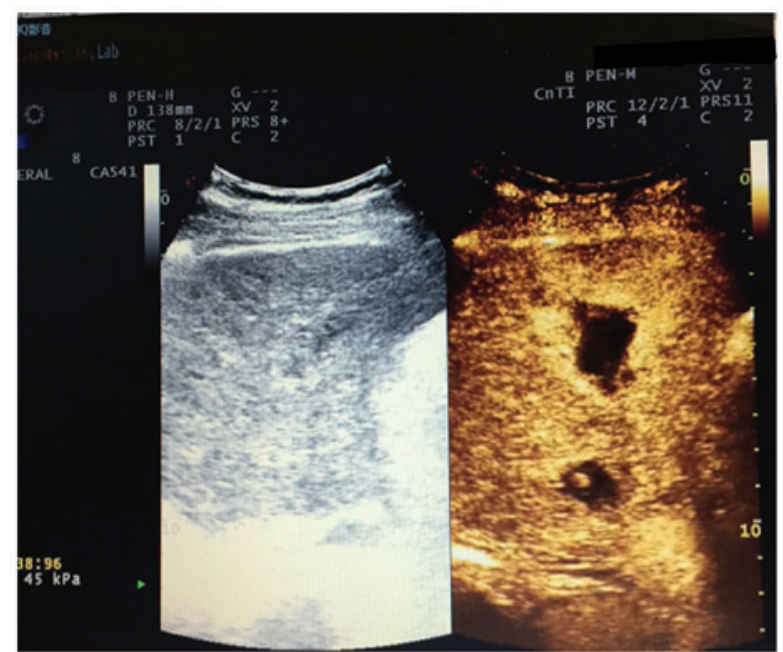

Figure 2. Contrast-enhanced ultrasound image for small hepatocellular carcinoma after treatment in the radiofrequency group.

them and killing cancer tissues. On the other hand, RFA can increase the number of local immune cells in the tumor and tumor cells in peripheral blood of the tumor, thereby reducing the metastasis of cancer cells. Implementation of ablation treatment against HCC should be achieved based on imaging methods, which primarily include ultrasound, magnetic resonance, and computed tomography (12-15). In terms of the implementation of RFA under ultrasonic labeling in the treatment of patients with HCC disease, previous findings have confirmed that this method has many advantages such as no radiation on the human body, simple operation, and the ability to observe lesions in real time. However, in contrast to an ultrasonic image cannot be formed due to small liver lesions, no vessel violations and iso-echoic HCC, ordinary ultrasound labeling is inadequate as it is difficult to accurately locate the cancer tissue (16-18). Consequently, during the treatment of HCC by RFA, CEUS technology should be employed as it enhances image sharpness and contrast, detects enhanced blood flow data and the lesion infiltration range, which cannot 


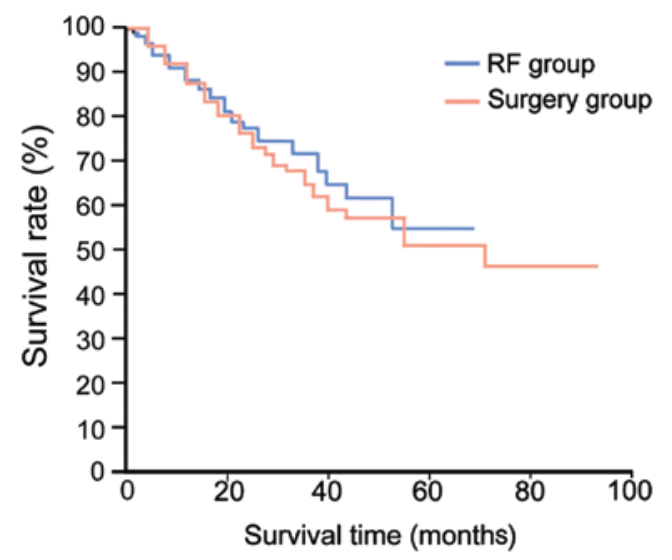

Figure 3. Comparison of survival rates between the surgery and radiofrequency (RF) groups.

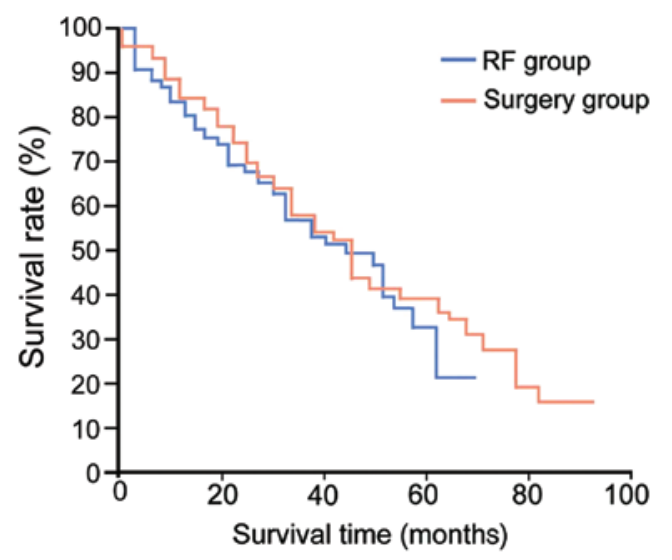

Figure 4. Comparison of tumor-free survival rates between the surgery and radiofrequency $(\mathrm{RF})$ groups.

be identified by ordinary ultrasound, thereby indicating the efficacy for more thorough treatment by RFA. Previously, it was reported that CEUS technology increased the clinical diagnostic rate of small HCC from 53 to 94\% (19-21).

Targeted treatment of cancer is an emerging type of technology together with the development of molecular and genetic engineering. The application of such technology can improve the survival rate of cancer patients by 5 years, and the disease progression of the cancer patients, and significantly improve the quality of life of the patients, thereby improving the survival time of patients with tumor under palliative treatment. Due to the above advantages, it has become a focus and hot spot in recent years in cancer clinical treatment $(22,23)$. The multi-kinase inhibitor sorafenib (Nexavar) is an oral drug and a multi-target signal transduction inhibitor, which can block the growth and proliferation of a variety of tumor cells, inhibit the growth of tumor microvessels and induce tumor cell apoptosis simultaneously, with improved antitumor activity $(24,25)$. Sorafenib is the first molecular-targeted drug currently achieving a good treatment effect in the clinical treatment of HCC (26). Combined sorafenib and RFA in the treatment of early small $\mathrm{HCC}$ can play their respective efficacy of RFA and sorafenib. The sorafenib mechanism for the treatment of early small HCC may on the one hand, be that the cancer tissue growth can be inhibited by inhibiting the signal transduction pathway. On the other hand, the antitumor role can be exerted by inhibiting the biological activity of several varieties of tyrosinase receptors closely related to the growth of the new micro-vessel and tumor development (24-26).

Regarding the scheme of the combined sorafenib and RFA method and laparotomy resection in the treatment of early small HCC in the present study, the implementation of laparotomy resection was successful in treating the cancer lesions (100\%), although the bleeding volume during the laparotomy process was relatively larger, and the presence of hepatic portal obstruction led to relatively large trauma and other defects to the patients. Simultaneously, the combined sorafenib and RFA treatment method has many advantages, such as simple operation, minimally invasive operation and small invasion, but also the defects of the residual lesions after ablation. To this end, the comparison of the combined sorafenib and RFA method and laparotomy resection method in the treatment of early small HCC should be assessed mainly based on the postoperative long-term prognosis. Hong et al (27) identified that the local recurrence rate of RFA in the treatment of small HCC was relatively higher, albeit there were no differences between the RFA method and laparotomy resection method in the distant lesion metastasis and overall survival rates. Ikeda et al (28) believed based on their studies that, in the course of the treatment of small HCC, by cost-benefit comparison, RFA had a better treatment effect compared to laparotomy resection, and that oral sorafenib as supplemental treatment after RFA treatment can significantly reduce the recurrence rate of small HCC.

The present findings have shown that after one month of treatment, the radical effective rate in the surgery group and RF group was $100 \%$. Contrast-enhanced ultrasound images were used to obtain the result for patients in the RF group. During the 5-year follow-up, the tumor recurrence rate in the surgery group was $18.3 \%$, significantly lower than the tumor recurrence rate in the RF group at $38.3 \%(\mathrm{P}<0.05)$. The occurrence rates of postoperative pain, fever, abdominal bleeding, infection and other complications in the surgery group were significantly higher than the complication occurrence rate $(\mathrm{P}<0.05)$ of patients in the RF group. The average survival time of the patients in the surgery group was $51.2 \pm 1.5$ months, the survival rates during the first, third and fifth year were $90.7,71.5$ and $56.7 \%$, respectively. The average survival time of the patients in the RF group was $64.6 \pm 2.4$ months, and the survival rates during the first, third and fifth year were $91.1,72.8$ and $57.5 \%$, respectively. The difference between the two groups was not statistically significant. The tumor-free survival rates in the surgery group during the first, third and fifth year were $87.8,44.3$ and $33.2 \%$, respectively. The tumor-free survival rates in the RF group during the first, third and fifth year were 86.2, 48.3 and $34.6 \%$, respectively. The difference between the two groups was not statistically significant $(\mathrm{P}<0.05)$. Some studies $(29,30)$ suggested that, in terms of the overall survival rate and disease-free survival rate, the clinical effect of combined sorafenib and RFA was equivalent to that of the laparotomy resection in the treatment, simultaneously the former's recurrence rate was higher, albeit the latter had more complications. The conclusions of the present study are more consistent with the perspectives of Hong et al (27) and Ikeda et al (28) as described above. 
In summary, the combined sorafenib and RFA method and laparotomy resection method have their advantages in the treatment of early small HCC, under the permissible hospital medical conditions, with the former method being capable of partially replacing the latter method and being used as a preferred treatment means in the treatment of early small HCC.

\section{Acknowledgements}

The present study was supported by the National Natural Science Foundation of China (nos. 81071888 and 81070344; 81000173, 81070322 and 81270491); the National Key Basic Research project, no. 2012CB517501; the 100-Talents Program of the Shanghai Municipal Health Bureau, (no. XBR2011007); the Cross Research Fund of Shanghai Jiaotong University Biomedical Engineering (no. YG2012MS37); the Shanghai Natural Science Foundation (no. 13ZR1426700); and Xinhua Hospital Clinical Research project (no. 15LC16).

\section{References}

1. Dy GK, Hillman SL, Rowland KM Jr, Molina JR, Steen PD, Wender DB, Nair S, Mandrekar S, Schild SE and Adjei AA; North Central Cancer Treatment Group Study N0326: A front-line window of opportunity phase 2 study of sorafenib in patients with advanced nonsmall cell lung cancer: North Central Cancer Treatment Group Study N0326. Cancer 116: 5686-5693, 2010

2. Cabibbo G and Craxi A: Epidemiology, risk factors and surveillance of hepatocellular carcinoma. Eur Rev Med Pharmacol Sci 14: 352-355, 2010.

3. Peng L, Zhou Y, Ye X and Zhao Q: Treatment-related fatigue with everolimus and temsirolimus in patients with cancer - a meta-analysis of clinical trials. Tumour Biol 36: 643-654, 2015.

4. Cheng AL, Kang YK, Chen Z, Tsao CJ, Qin S, Kim JS, Luo R, Feng J, Ye S, Yang TS, et al: Efficacy and safety of sorafenib in patients in the Asia-Pacific region with advanced hepatocellular carcinoma: A phase III randomised, double-blind, placebo-controlled trial. Lancet Oncol 10: 25-34, 2009

5. Cucchetti A, Piscaglia F, Cescon M, Serra C, Colecchia A, Maroni L, Venerandi L, Ercolani G and Pinna AD: An explorative data-analysis to support the choice between hepatic resection and radiofrequency ablation in the treatment of hepatocellular carcinoma. Dig Liver Dis 46: 257-263, 2014.

6. Zhai B and Sun XY: Mechanisms of resistance to sorafenib and the corresponding strategies in hepatocellular carcinoma. World J Hepatol 5: 345-352, 2013 .

7. Zhao X, Tian C, Puszyk WM, Ogunwobi OO, Cao M, Wang T, Cabrera R, Nelson DR and Liu C: OPA1 downregulation is involved in sorafenib-induced apoptosis in hepatocellular carcinoma. Lab Invest 93: 8-19, 2013.

8. European Association For The Study Of The Liver; European Organisation For Research and Treatment of Cancer: EASL-EORTC clinical practice guidelines: Management of hepatocellular carcinoma. J Hepatol 56: 908-943, 2012.

9. Gravante G, Overton J, Sorge R, Bhardwaj N, Metcalfe MS, Lloyd DM and Dennison AR: Radiofrequency ablation versus resection for liver tumours: An evidence-based approach to retrospective comparative studies. J Gastrointest Surg 15: 378-387, 2011.

10. Bruix J and Sherman M; American Association for the Study of Liver Diseases: Management of hepatocellular carcinoma: An update. Hepatology 53: 1020-1022, 2011.

11. Bhardwaj N, Strickland AD, Ahmad F, El-Abassy M, Morgan B, Robertson GS and Lloyd DM: Microwave ablation for unresectable hepatic tumours: Clinical results using a novel microwave probe and generator. Eur J Surg Oncol 36: 264-268, 2010.

12. Sherman M, Burak K, Maroun J, Metrakos P, Knox JJ, Myers RP, Guindi M, Porter G, Kachura JR, Rasuli P, et al: Multidisciplinary Canadian consensus recommendations for the management and treatment of hepatocellular carcinoma. Curr Oncol 18: 228-240, 2011.
13. Yim HJ, Suh SJ and Um SH: Current management of hepatocellular carcinoma: An Eastern perspective. World J Gastroenterol 21: 3826-3842, 2015.

14. Ma H, Zhang Y, Wang Q, Li Y, He J, Wang H, Sun J, Pan K, Chen $\mathrm{M}$ and Xia J: Therapeutic safety and effects of adjuvant autologous RetroNectin activated killer cell immunotherapy for patients with primary hepatocellular carcinoma after radiofrequency ablation. Cancer Biol Ther 9: 903-907, 2010.

15. Karabulut K, Aucejo F, Akyildiz HY, Siperstein A and Berber E: Resection and radiofrequency ablation in the treatment of hepatocellular carcinoma: A single-center experience. Surg Endosc 26: 990-997, 2012.

16. Birsen O, Aliyev S, Aksoy E, Taskin HE, Akyuz M, Karabulut K, Siperstein A and Berber E: A critical analysis of postoperative morbidity and mortality after laparoscopic radiofrequency ablation of liver tumors. Ann Surg Oncol 21: 1834-1840, 2014

17. Muallem N and Solomon SB: Advances in interventional oncology: Percutaneous therapies. Curr Radiol Rep 2: 52 , 2014.

18. Morimoto N, Isoda N, Watanabe S, Otake T, Hirosawa T, Tsukui M, Miyata N, Murayama K, Iwashita C, Takaoka Y, et al: A case of small hepatocellular carcinoma treated with laparoscopic multipolar radiofrequency ablation with a no-touch ablation procedure. Clin J Gastroenterol 7: 510-515, 2014.

19. Tsuji K, Yamazaki H, Nagai K, Matsui T, Tomonari A, Kang J, Sakurai Y, Kodama Y and Maguchi H: A case of laparoscopic radiofrequency ablation therapy using bipolar RFA system for hepatocellular carcinoma. Kanzo 54: 819-825, 2013.

20. Kawamura Y, Ikeda K, Fukushima T, Hara T, Hosaka T, Kobayashi M, Saitoh S, Sezaki H, Akuta N, Suzuki F, et al: Potential of a no-touch pincer ablation procedure for small hepatocellular carcinoma that uses a multipolar radiofrequency ablation system: An experimental animal study. Hepatol Res 44: 1234-1240, 2014

21. Forner A, Reig ME, de Lope CR and Bruix J: Current strategy for staging and treatment: The BCLC update and future prospects. Semin Liver Dis 30: 61-74, 2010.

22. Soerjomataram I, Lortet-Tieulent J, Parkin DM, Ferlay J, Mathers C, Forman D and Bray F: Global burden of cancer in 2008: A systematic analysis of disability-adjusted life-years in 12 world regions. Lancet 380: 1840-1850, 2012.

23. Li L, Zhang J, Liu X, Li X, Jiao B and Kang T: Clinical outcomes of radiofrequency ablation and surgical resection for small hepatocellular carcinoma: A meta-analysis. J Gastroenterol Hepatol 27: 51-58, 2012.

24. Kharaziha P, De Raeve H, Fristedt C, Li Q, Gruber A, Johnsson P, Kokaraki G, Panzar M, Laane E, Osterborg A, Zhivotovsky B, Jernberg-Wiklund H, Grander D, Celsing F, Bjorkholm M, Vanderkerken K and Panaretakis T: Sorafenib has potent antitumor activity against multiple myeloma in vitro, ex vivo, and in vivo in the 5T33MM mouse model. Cancer Res 72: 5348-5362, 2012.

25. Tamaskar I, Garcia JA, Elson P, Wood L, Mekhail T, Dreicer R, Rini BI and Bukowski RM: Antitumor effects of sunitinib or sorafenib in patients with metastatic renal cell carcinoma who received prior antiangiogenic therapy. J Urol 179: 81-86, 2008.

26. Waghray A, Balci B, El-Gazzaz G, Kim R, Pelley R, Narayanan MK, Estfan B, Romero-Marrero C, Aucejo F: Safety and efficacy of sorafenib for the treatment of recurrent hepatocellular carcinoma after liver transplantation. CLIN TRANSPLANT 2013;27:555-561

27. Hong SN, Lee SY, Choi MS, Lee JH, Koh KC, Paik SW, Yoo BC, Rhee JC, Choi D, Lim HK, et al: Comparing the outcomes of radiofrequency ablation and surgery in patients with a single small hepatocellular carcinoma and well-preserved hepatic function. J Clin Gastroenterol 39: 247-252, 2005.

28. Ikeda K, Kobayashi M, Saitoh S, Someya T, Hosaka T, Sezaki H, Suzuki Y, Suzuki F, Akuta N and Arase Y: Cost-effectiveness of radiofrequency ablation and surgical therapy for small hepatocellular carcinoma of $3 \mathrm{~cm}$ or less in diameter. Hepatol Res 33: 241-249, 2005.

29. Feng X, Xu R, Du X, Dou K, Qin X, Xu J, Jia W, Wang Z, Zhao H, Yang S, Guo C, Liu T and Ma K: Combination therapy with sorafenib and radiofrequency ablation for BCLC Stage 0-B1 hepatocellular carcinoma: A multicenter retrospective cohort study. Am J Gastroenterol 109: 1891-1899, 2014.

30. Berretta M, Di Francia R and Tirelli U: Sorafenib combined with percutaneous radiofrequency ablation for the treatment of medium-sized hepatocellular carcinoma. Eur Rev Med Pharmacol Sci 19: 2521-2522, 2015. 